Relations industrielles

Industrial Relations

\title{
Action syndicale et Bien commun
}

\section{Roger Chartier}

Volume 15, numéro 4, octobre 1960

URI : https://id.erudit.org/iderudit/1021942ar

DOI : https://doi.org/10.7202/1021942ar

Aller au sommaire du numéro

Éditeur(s)

Département des relations industrielles de l'Université Laval

ISSN

0034-379X (imprimé)

1703-8138 (numérique)

Découvrir la revue

Citer ce document

Chartier, R. (1960). Action syndicale et Bien commun. Relations industrielles /

Industrial Relations, 15(4), 483-487. https://doi.org/10.7202/1021942ar

Tous droits réservés (C Département des relations industrielles de l’Université Laval, 1960
Ce document est protégé par la loi sur le droit d'auteur. L'utilisation des services d'Érudit (y compris la reproduction) est assujettie à sa politique d'utilisation que vous pouvez consulter en ligne.

https://apropos.erudit.org/fr/usagers/politique-dutilisation/ 
sabilité en les associant aux décisions d'ordre économique et social, bref de leur faire jouer, là où ils sont en mesure de le faire, le rôle d'organes de gouvernement de la vie sociale.

Disons, en passant, qu'il s'agit bien de la «vie sociale » et non de la vie politique, car les deux se distinguent, et il serait dangereux de les confondre. Quand l'Eglise réclame l'organisation professionnelle, c'est la vie économique et sociale qu'elle demande d'organiser; elle veut des organes économiques et sociaux, non des organes politiques.

Dans le domaine propre de l'économie, il faut reconnaître que les syndicats, tant de patrons que d'ouvriers, organisent plutôt des classes que la profession ellemême, des intérêts privés qu'un service public. Mais une collaboration organique s'amorce entre tous les agents de la production; l'Eglise y applaudit. Selon le mot de S. Em. le cardinal Léger, elle soutient qu'«une saine économie doit être organique ». Ce n'est pas de se déduire que l'Eglise demande aux syndicats, c'est de construire ensemble un ordre social juste, un ordre centré sur leur collaboration, qui fasse de l'homme le sujet et la fin de l'économie.

Il y a donc concordance foncière entre les aspirations de notre monde actuel à un ordre social plus juste et plus humain, et les enseignements et les directives que nous donne l'Eglise sur les moyens à prendre pour réaliser un tel ordre. Il ne s'agit pas simplement d'élever des structures ou même d'agencer des institutions, il s'agit encore bien plus de donner à l'ensemble un esprit, un esprit qui, en inculquant à chacun, aux groupes comme aux individus, le sens du dévouement, de la collaboration et même du sacrifice, fasse fonctionner le tout aussi harmonieusement qu'un corps humain en bonne santé. C'est là notre tâche à nous, chrétiens catholiques, de donner cet esprit, cette doctrine dont notre monde a un si pressant besoin, car e ce qu'est l'âme dans le corps, voici ce que sont, dans le monde, les chrétiens ».

\section{ACTION SYNDICALE et BIEN COMMUN}

\section{Roger Chartier}

\section{LE BIEN COMMUN}

Le bien est ce que tout être désire en tant qu'il désire sa perfection, puisque celle-ci est pour chaque ètre son bien.

Le bien est conmun dès qu'il cesse d'être le bien d'un seul individu, qu'il est partagé par plusieurs. Le bien commun appartient donc à tous les membres d'une collectivité. Ce bien commun sera particulier si la collectivité en cause est un corps intermédiaire entre l'individu et la société globale (une ville, une association religieuse, un groupement commercial, un club social, un syndicat, une fédération ou une centrale syndicale, et ainsi de suite); il sera aussi particulier s'il est le bien commun d'une province ou d'un Etat au sein d'un pays, ou encore le bien commun d'un pays au sein de la vaste société des nations. 
Ce bien commun sera général s'il est la propriété collective de tous les membres d'une société politique donnée (par exemple, la province de Québec, le Canada) prise comme un tout ultime, c'est-à-dire sans référence à un cadre politique plus vaste.

Enfin, dernière distinction, le bien commun d'une province, d'un pays ou du concert des nations est un bien public; celui d'un corps intermédiaire est un bien privé.

Le bien commun général, on le conçoit, dépasse de cent coudées le niveau des intérêts des groupes particuliers, comme le bien de ces derniers transcende les intérêts des individus-membres. Le bien commun d'un groupe n'est pas la somme arithmétique des biens des individus qui le composent, pas plus que le bien de la société civile n'est constitué par l'addition des biens particuliers, qu'ils soient individuels ou collectifs.

Et pourtant, le bien commun général s'étend davantage au particulier (groupe ou individu) que le bien particulier lui-même. Dès lors, selon l'expression justement paradoxale de M. Charles de Koninck (De la primauté du bien commun, p. 9),

Le bien commun n'est pas un bien qui ne serait pas le bien des particuliers, et qui ne serait que le bien de la collectivité envisagée comme une sorte de singulier... Quand nous distinguons le bien commun du bien particulier, nous n'entendons pas par là qu'il n'est pas le bien des particuliers: s'il n'était pas le bien des particuliers, il ne serait pas vraiment commun.

Le bien commun n'est donc pas pour l'individu un bien étranger, mais un bien propre. Le bien commun général, en dépit de sa transcendance, n'est pas une réalité désincarnée et distante. Il n'est pas indépendant, loin de là, de l'orientation des biens particuliers vers tel ou tel équilibre, d'ailleurs instable. Ainsi, pour ne donner qu'un exemple, les efforts du syndicalisme ouvrier ont certes mené, sinon à une redéfinition du bien commun général, au moins à une nouvelle pondération relative de ses éléments constituants, de telle sorte qu'aujourd'hui il se peut qu'on attache plus d'importance aux valeurs de sécurité économico-sociale qu'à certaines valeurs, parfois un peu théoriques, de liberté individuelle. De plus, il est très normal que le bien commun reflète, en des aspects importants, les biens particuliers de tel ou tel groupe imposant (par sa force numérique, économique ou morale) de la société.

Ce bien commun, qui est le bien d'une communauté humaine, s'obtient par l'effort de tous pour tous. Il se choisit, se désire et se recherche en commun. Et, point très important, il se réalise, se déverse communément sur chacun des individus ou des groupes sociaux sans se diviser ni s'aliéner.

\section{ACtion SyNDICALE ET BIEN COMMUN: LES PERSPECTIVES}

a) Le syndicalisme est de bien commun: à ce titre, il commanderait, chez les employeurs comme dans le grand public, un accueil sincère et des attitudes favorables. Le législateur a compris la fonction éminente et nécessaire du syndicalisme . ouvrier en faisant de la convention collective un contrat d'ordre mi-public, mi- 
privé, en édictant que le syndicat groupant la majorité absolue des employés d'une unité de négociation donnée parle au nom de tous les employés en cause, qu'il peut exiger un dialogue de bonne foi avec l'employeur en vue de la conclusion d'une convention collective qui produira ses effets sur tous les employés de l'unité de négociation en question.

b) Le syndicalisme sert le bien commun: voici une seconde perspective, d'ailleurs fort légitime; ici, on fait le bilan de l'activité du syndicalisme en regard d'un bien commun général plus ou moins clairement défini: on précise les efforts fructueux du syndicalisme sur la législation sociale, dans le domaine de la culture et plus particulièrement au plan de la négociation collective, expression et cadre de la \& démocratie industrielle».

\section{c) L'action syndicale doit tendre vers le bien commun:}

Une troisième perspective s'exprime par l'affirmation globale que le syndicalisme ouvrier, dans toute son activité, doit être en tension vers le bien commun. Telle quelle, cette position normative appelle des éclaircissements fondamentaux. Et d'abord, de quel bien commun s'agit-il? S'il est question du bien commun particulier du corps intermédiaire appelé syndicalisme, nous en sommes. Mais encore faudrait-il distinguer entre le syndicat local et l'union ou fédération, entre celle-ci et la centrale syndicale, et enfin entre telle centrale et telle autre. Aux divers paliers des structures syndicales se situent des groupes divers, dont les biens particuliers sont partiellement différents et par certains aspects divergents les uns des autres.

C'est donc entendu: les groupements syndicaux et les individus en leur sein poursuivent, par leur action économique, sociale et morale, leurs biens particuliers. Allons plus loin: ces biens particuliers (biens des membres et biens de l'institution) sont les seuls que les groupements syndicaux (comme tous les autres corps intermédiaires d'ailleurs) aient le devoir et la faculté de poursuivre directement. Et on a par ricochet le devoir de les juger sévèrement s'ils s'éloignent de la poursuite de tels biens particuliers spécifiques: voilà le critère, voilà la norme.

Mais, remarquera-t-on, que devient dans tout ceci le bien commun général ! N'est-ce pas inviter individus et groupes à tous les égoïsmes que de leur imposer comme norme concrète d'action le seul bien particulier qui les concerne ? Que non pas! Nous avons vu précédemment combien peuvent être étroites, surtout dans un contexte démocratique, les relations entre bien commun particulier et bien commun général. De plus, nous nous hâtons d'ajouter que si le bien commun général ne peut ni ne doit servir de guide direct pour l'action des individus et des corps intermédiaires, ces derniers doivent quand même en tenir compte comme d'une donnée de base dans la mesure où il est concrètement défini, ne jamais agir à son encontre quand il est clair et respecter les biens particuliers d'autres individus et groupes au sein de la société globale.

Il faut au plus tôt déduire l'équivoque selon laquelle la poursuite vigoureuse des intérêts particuliers est une hérésie sociale. Il importe au plus vite de libérer les corps intermédiaires comme le syndicalisme à tous ses paliers du jugementmassue par lequel on condamne ou on appuie leur action au nom d'un certain bien général le plus souvent imprécis. 
Ce qu'il faut bien voir, c'est que les structures juridico-économique présentes, chez nous, ne se prêtent que très mal à l'utilisation du bien commun général comme critère d'évaluation de l'activité syndicale. Nous vivons en régime de libre entreprise et de syndicalisme libre, celui-ci s'adaptant à celle-là et édifiant ses cadres aux dimensions de celle-là. Dans ce contexte, c'est le choc de divers biens particuliers qui aboutira, par divers compromis mutuellement acceptables, à une certaine définition opérationnelle du bien commun dans l'industrie. De plus, comme nous le soulignerons davantage plus loin, notre législation du travail condamne la négociation collective à la courte vue en limitant le cadre du dialogue aux dimensions de l'usine locale.

Parallèlement aux structures, les attitudes de beaucoup de gens sont hostiles à l'élargissement des vues et du champ d'action des syndicats. D'une part, ce sont justement ces mêmes personnes qui entendent faire la comptabilité des gestes syndicaux sur la base de leur approximation du bien commun général, qui invitent le syndicalisme ouvrier à la collaboration aux dépens de la revendication «outrée 》, bref, qui exhortent les syndicats à «mûrir 》, à voir grand, large et désintéressé. Et d'autre part, du même souffle, ces personnes s'inscriront en faux contre toute forme d'action politique par le syndicalisme, refusant de reconnaître l'apport passé de ce dernier en matière de législation sociale, de faire les distinctions nécessaires entre l'action politique indirecte (éducative) et directe (partisane ou non partisane), et de voir la nécessité pour l'action syndicale de dépasser le cadre strict de la négociation collective. Et même à ce dernier niveau, on refusera d'épauler l'organisme syndical qui lutte pour élargir la négociation collective aux dimensions de l'entreprise à plusieurs usines syndiquées, sans parler de l'industrie et de l'ensemble de l'économie. Ne serait-ce pas là une occasion merveilleuse d'assurer la promotion concrète de l'\& organisation professionnelle » et de la «démocratisation de l'économie»?

Dans la conjoncture actuelle, c'est à l'Etat et à l'Etat seul, qu'il incombe de définir avec le plus de clarté possible les impératifs du bien commun général quand la nécessité s'en fait sentir; c'est à lui qu'il revient de s'en faire le défenseur et le promoteur contre les poussées égoïstes de certains groupes d'intérêts et dans la ligne des voeux profonds de la majorité du peuple. L'Etat devra s'aider, dans ses définitions et dans ses orientations, des lumières des divers groupes économicosociaux; mais en dernière analyse, c'est lui le pilote et le metteur d'ordre, qui doit dire le juste et le bien quand ce dernier ne ressort pas clairement de la libre activité des individus et des groupes intermédiaires. Selon la formule de Pie XI, il appartient exclusivement à l'Etat de «diriger, surveiller, stimuler, contenir selon que le comportent les circonstances ou l'exige la nécessité 》(Quadragesimo Anno).

Il existe évidemment des parties d'alternative à la conjoncture juridique présente. Ainsi, le syndicalisme pourrait être appelé à participer de plus en plus étroitement à la fonction de l'Etat; on peut concevoir un syndicalisme qui ne serait que le prolongement de l'Etat dans la vie économique. Entre le syndicalisme d'Etat et le syndicalisme totalement libre, toute une gamme de degrés sont possibles. Le syndicalisme d'Etat aurait évidemment le devoir de centrer son action plus directement sur le bien commun général. Reste à savoir si on souhaite vraiment l'avènement chez nous de cette sorte de syndicalisme. 
De plus, on pourrait permettre la négociation à l'échelle de l'entreprise (à usines multiples) et de l'industrie. Ce serait une très bonne façon d'imposer aux négociateurs, des deux côtés de la table, des perspectives plus vastes et un souci plus réel des conséquences de leurs gestes sur l'économie générale.

Bref, quand on affirme que l'action syndicale doit tendre vers le bien commun, il faut y mettre des nuances d'une extrême importance. D'ailleurs, l'étude concrète d'une réalité industrielle terriblement complexe suggère d'elle-même le sens des distinctions, le réalisme des faits et l'humilité des assertions.

\section{POSSIBILITÉS ET CONDITIONS DE COLLABORATION ENTRE LES AGENTS DE L'ÉCONOMIE}

Jean-Réal Cardin

\section{LES BASES DE LA COLLABORATION}

La collaboration entre les agents de l'économie est nécessairement conditionnée par le contexte institutionnel dans lequel elle est appelée à s'exprimer. Quelles sont les lignes de force de ce contexte? A la base, nous avons la liberté d'entreprise. Cette liberté s'accompagne sur le plan juridique de la liberté de contrat. Nous sommes encore en ce qu'on appelle le régime de salariat. Viennent s'ajouter ensuite la liberté dans le choix des professions, des métiers et des occupations, laquelle emporte comme corrollaires nécessaires le droit à l'association et à l'action collective sur le plan des intérêts communs dans l'exercice de ces métiers et professions, (le droit de grève ou de contre-grève, par exemple, en matière de relations du travail). Ces libertés, comme les autres qui ont été énumérées plus haut, ne sont pas absolues, cela va de soi, mais elles n'en appartiennent pas moins au patrimoine commun des sociétés libres dont nous nous réclamons.

Notre système économique est de plus en plus structuré et des groupes d'intérêts coexistent et se partagent le pouvoir en matière de décisions économiques et sociales. Cette dernière constatation emporte deux conséquences majeures:

Premièrement, les relations industrielles sont de plus en plus des relations collectives, des relations de groupes et l'équilibre résultant de l'exercice simultané des différentes libertés auxquelles nous avons fait allusion il y a un instant, s'établit non plus sur le plan des individus (patron-ouvrier, par exemple) mais davantage sur celui des groupes organisés au moyen de ce qu'on appelle la négociation collective.

Deuxièmement, étant donné la présence de différents groupes d'intérêts se partageant le pouvoir en matière de décisions économiques et les conflits inévitables qui en résultent, l'Etat, en tant que représentant l'ensemble de la communauté possède un certain droit d'intervention fondé à la fois sur les attributs qu'on lui concède en démocratie et sur les exigences que commandent à un certain moment les degrés respectifs de pouvoir entre ces différents groupes économiques. 